GEOLOGICAL SURVEY CIRCULAR 716

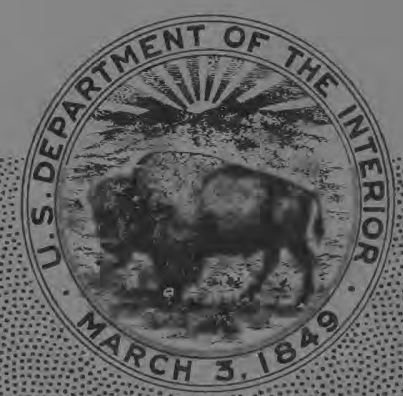

Worldwide Directory of National Earth-Science Agencies 


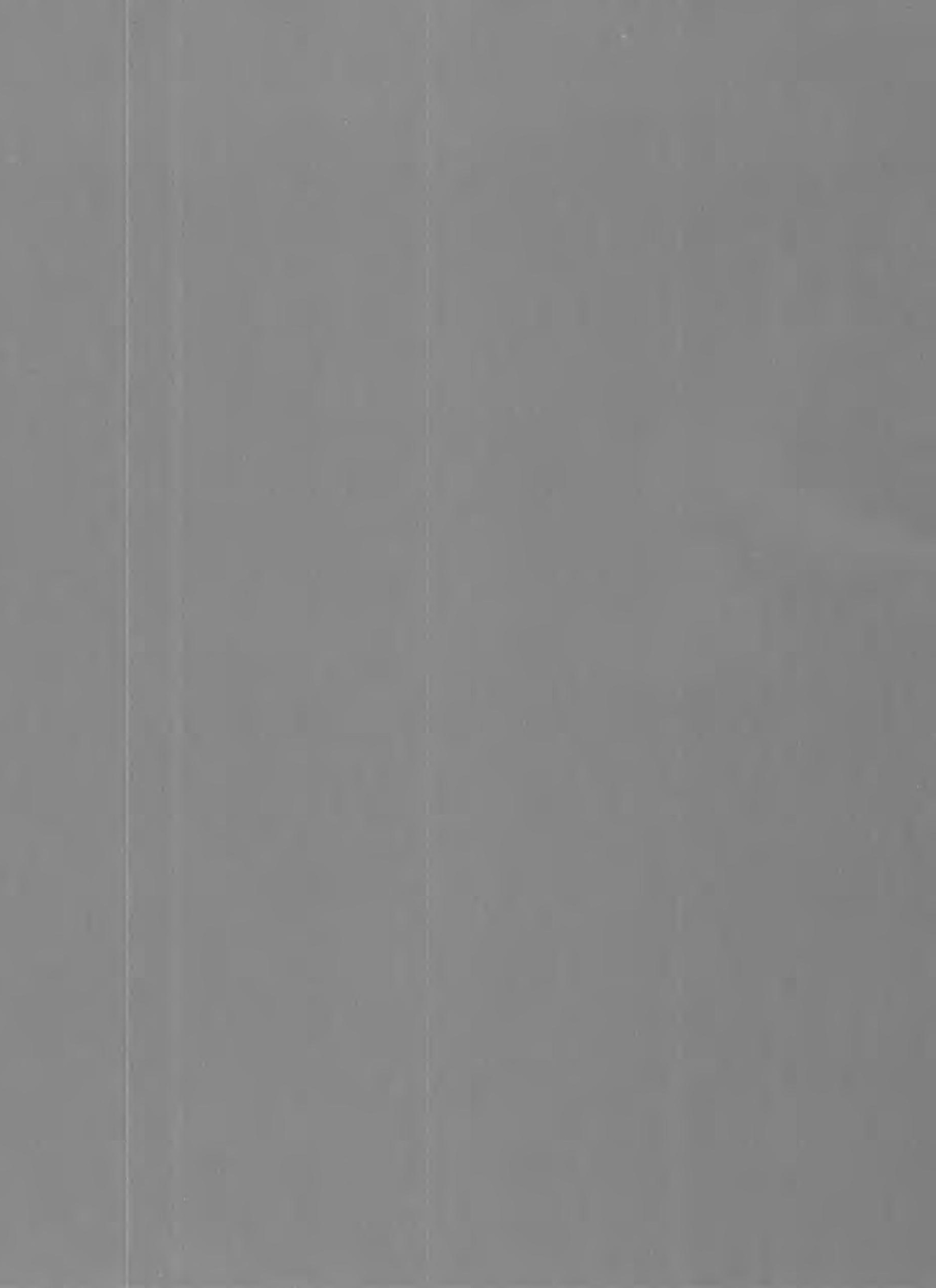




\title{
Worldwide Directory of National Earth-Science Agencies
}

\author{
Compiled by Anne Lucas Falk and \\ Ralph L. Miller
}

GEOLOGICAL SURVEY CIRCULAR 716

A listing of goternmental carth-science

orsanizations whose functions are similar

to those of the I'.S. Geological Surr' ')' 
United States Department of the Interior

ROGERS C. B. MORTON, Secretary

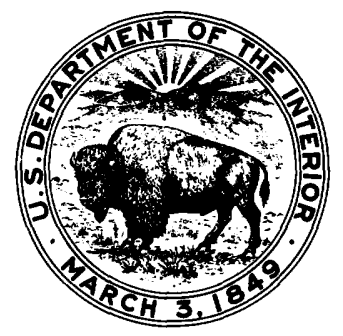

Geological Survey

V. E. McKelvey, Director 


\section{CONTENTS}

Introduction -

North America

Canada

Greenland

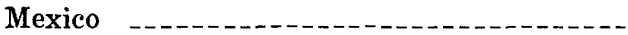

United States

Caribbean islands

Barbados

Cuba

Dominican Republic -.....

Guadeloupe and Dependencies

Haiti

Jamaica -

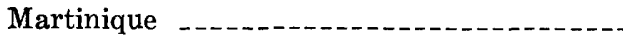

Puerto Rico -

Trinidad and Tobago

Central America

Costa Rica

El Salvador

Guatemala

Honduras

Nicaragua -

Panama _.

South America _.

Argentina

Brazil

Chile --.--_-

Colombia

Ecuador

French Guiana

Guyana -

Netherlands Antilles -.-_-

Paraguay

Peru

Surinam

Uruguay

Venezuela

Europe

$\begin{array}{ll}\text { Albania } & 8 \\ \text { Austria } & 8 \\ \text { Belgium } & 8 \\ \text { Bulgaria } & 9 \\ \text { Czechoslovakia } & 9 \\ \text { Fenmark } & 9 \\ \text { France } & 9 \\ \text { Federal Republic of Germany (West) } & 10 \\ \text { German Democratic Republic (East) } & 10 \\ \text { Great Britain } & \\ \text { Greece - } & \\ \text { Hungary - } & \end{array}$
Page

1 Europe-Continued

2

2

2

2

3

3

3

3

3

3

3
3

3

4

\section{4}

\section{4}

4

4

\section{4}

Iceland

Ireland 11

Italy _._.

Liechtenstein _._. 12

Luxembourg _..._- 12

Malta

Netherlands _......... 12

Northern Ireland .

Norway _.............. 12

Poland _.

Portugal _-

Romania -

Spain _._. 14

Sweden _._.

Switzerland _._._._._._._. 14

Union of Soviet Socialist Republics (USSR) 15

Yugoslavia _..._. 16

Africa _... 16

Algeria _._. 16

Angola _.

Botswana _... 16

Burundi _... 16

Cameroon _..._. 16

Central African Republic _........ 17

Chad _.... 17

Congo, Republic of _..._. 17

Dahomey _... 17

Ethiopia -.... 17

French Territory of Afars and Issas _.... 18

Gabon -

Gambia -

Ghana -

Guinea -

Ivory Coast

Kenya _... 18

Lesotho _._-_._- 19

Liberia _.

Libya _...

Malagasy Republic _-_._. 19

Malawi _._. 19

Mali _-_._. 19

Mauritania _...... 20

Mauritius _...- 20

Morocco _.

Mozambique -

Niger -

Nigeria -......... 20

Portuguese Guinea

Reunion _- 21

Rhodesia _....... 21

Rwanda _... 21

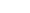

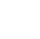

(1)

(3)

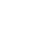

.

4

.

6

6

6

(6

(16

17

7

17

7
18

8


Africa-Continued

Senegal

Sierra Leone _.

Somalia -..-

South Africa -

South-West Africa (Namibia)

Spanish Sahara

Sudan

Swaziland

Tanzania

Togo

Tunisia

Uganda

United Arab Republic

Upper Volta

Zaire

Zambia

Near East-South Asia

Afghanistan

Bangladesh

Cyprus

India

Iran

Iraq

Israel

Jordan

Kuwait

Lebanon

Nepal

Oman

Pakistan

Qatar

Saudi Arabia
Near East-South Asia-Continued

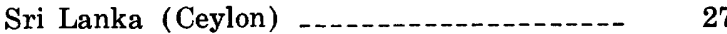

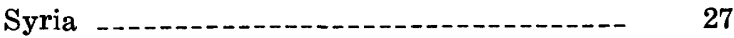

Turkey -

Yemen

East Asia-Pacific Region -_._._._._. 27

Australia _._.

Borneo _................ 28

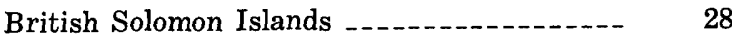

Brunei

Burma -

Peoples Republic of China -...-_..- 28

Republic of China (Taiwan) -......... 28

Fiji

Hong Kong _.

Indonesia _......... 29

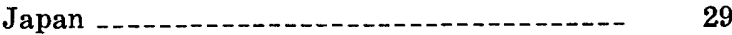

Khmer Republic (Cambodia) _.......... 30

Korea, Democratic People's Republic

(North) -

Korea, Republic of (South) -.. 30

Laos -

Malaysia _...

Mongolian People's Republic _._-_._-_._-_ 31

New Caledonia -........ 31

New Guinea -_._._. 31

New Hebrides -

New Zealand _... 31

Philippines - .

Singapore -

Thailand _..... 32

Democratic Republic of Vietnam (North) -- 32

Republic of Vietnam (South) _-_-_-_-_- $\quad 32$

Western Samoa -.......- 32 


\title{
WORLDWIDE DIRECTORY OF NATIONAL EARTH-SCIENCE AGENCIES
}

\author{
Compiled by Anne Lucas Falk and Ralph L. Miller
}

\section{INTRODUCTION}

This directory was originally planned and prepared for internal useto provide the Geological Survey (USGS) with a ready reference to earthscience bureaus around the world that have functions similar to those of one or more of the operating divisions of the USGS. Experience has indicated, however, that the directory fills a much broader need; hence, it has been updated and enlarged for publication. The compilers recognize that many more earth-science organizations than are listed here are doing work similar to that of the USGS. To keep the directory to a convenient size, however, only governmental institutions have been listed for each country; of these, we include only one, or at most a very few, of those whose functions match or overlap those of one or more divisions of the USGS.

Wherever feasible, names of agencies in countries whose languages are based primarily on the Latin alphabet are given in the language of the country. English translations have been added in brackets where they seem necessary for easy understanding. Names of agencies in countries whose languages utilize an alphabet other than the Latin are given in English.

The principal functions of the USGS and of the agencies in the directory are indicated by the following code:

$$
\begin{aligned}
& \mathrm{G}=\text { Geology } \\
& \mathrm{C}=\text { Cartography } \\
& \mathrm{H}=\text { Hydrology } \\
& \mathrm{R}=\text { Minerals and petroleum regulation }
\end{aligned}
$$

This directory is based on information available to the compilers in the summer of 1974. It undoubtedly has some errors and some serious omissions. In addition, some changes will have taken place since it was compiled. The compilers will appreciate having such changes or errors called to their attention by users. 


\section{DIRECTORY}

NORTH AMERICA

\section{Canada}

G Geological Survey of Canada

Department of Energy, Mines, and Resources

601 Booth Street

Ottawa, Ontario, K1A 0E8

Director: D. J. McLaren

G Canada Centre for Mineral and Energy Technology

Department of Energy, Mines, and Resources

588 Booth Street

Ottawa, Ontario, K1A 0E4

Director: D. F. Coates

C Surveying and Mapping Branch

Department of Energy, Mines, and Resources

601 Booth Street

Ottawa, Ontario, K1A 0E9

Director: George Zarzycki

$\mathrm{H} \quad$ Inland Waters Directorate

Department of Energy, Mines, and

Resources

601 Booth Street

Ottawa, Ontario, K1A 0E9

Director: A. T. Price

R National Energy Board

Place de Ville

112 Kent Street

Ottawa, Ontario, K1A 0E5

Chairman: M. A. Crowe

R Mineral Resources Branch

Department of Energy, Mines, and

Resources

601 Booth Street

Ottawa, Ontario, K1A 0E4

Director: W. K. Buck

\section{Greenland}

G Grønlands Geologiske Unders $\varnothing$ gelse

Østervoldgade 5-7, Tr. KL

DK-1350 København, Denmark

Director: K. Ellitsgaard-Rasmussen

\section{NORTH AMERICA-Continued}

Mexico

G Instituto de Geología

Universidad Nacional Autónoma de México

Ciudad Universitaria

México 20, D. F.

Director: Diego Córdoba

G Instituto de Geofísica

Universidad Nacional Autónoma de México

Ciudad Universitaria

México 20, D. F.

Director: Julian Adem

G Consejo de Recursos Naturales no Renovables

Niños Heroes 130

México 7, D.F.

Director General: Guillermo P. Salas

G Instituto de Biología (Includes Oceanography)

Universidad Nacional Autónoma de México

Ciudad Universitaria

México 20, D.F.

Director: Agustín Ayala Castañares

C, G Comisión de Estudios del Territorio Nacional (CETENAL)

San Antonio Abad 124, $5^{\circ}$ Piso

México 8, D.F.

Director: Juan B. Puig de la Parra

C Departmento Geográfico Militar

Secretaria de la Defensa Nacional

Lomas de Sotelo

Mexico 10, D.F.

Director: Joachim Orozco Camacho

H Secretaria de Recursos Hidráulicos

No. 69 Paseo de la Reforma

México 1, D.F.

Ministro: Leandro Rovirosa Wade 
NORTH AMERICA-Continued

\section{United States}

G, C, U. S. Geological Survey

$\mathrm{H}, \mathrm{R}$ National Center 12201 Sunrise Valley Drive

Reston, Virginia 22092

Director: V. E. McKelvey

G U.S. Bureau of Mines

Washington, D.C. 20240

Director: Thomas V. Falkie

\section{CARIBBEAN ISLANDS}

\section{Barbados}

G Bellairs Research Institute

St. James

President: Douglas R. Carter

C Ministry of Home Affairs

Government Headquarters

Bay Street

Bridgetown

Cuba

G Instituto de Geología

Academia de Ciencias de Cuba

Ave. Van-Troi no. 17203

Rancho Boyeros-Apartado Postal 10

La Habana

Director: Jesus F. de Albear

Franquiz

G Instituto Nacional de Recursos Minerales (IRCM)

O’Reilly y Aguacate

La Habana

Director: Salvador Salas

C Instituto Cubano de Cartografía y Catastro

Apartado Postal 2411

La Habana

H Instituto Nacional de Recursos Hidráulicos (INRH)

Humboldt 106 esp. P.

Vedado, La Habana

Director: Faustino Peréz

Hernández
CARIBBEAN ISLANDS-Continued

Dominican Republic

G Dirección de Minería

Secretaría de Estado de Fomento

Santo Domingo

G Instituto de Investigaciones

Geográficas y Geológicas

Universidad de Santo Domingo

Santo Domingo

C Instituto Geográfico Universitario

Calle El Conde No. 2

Santo Domingo

Director: José J. IIungría M.

C Instituto Cartográfico Militar de las Fuerzas Armadas

Santo Domingo

Director: José J. Hungría M.

\section{Guadeloupe and Dependencies}

G Arrondissement Mineralogique de la Guyane

B.P. 448

Pointe-a-Pitre

Guadeloupe

Haiti

G Service Géologique

Department of Agriculture, Natural Resources, and Rural Development

Damiens près Port-au-Prince

C Service de Géodesie et de Cartographie

Boulevard Harry Truman

Port-au-Prince

Director: Raymond B. Oriol

\section{Jamaica}

G Geological Survey Department

Hope Gardens

Kingston 6

C Survey Department

P.O. Box 493

Kingston

H National Water Authority

Kingston

Secretary-Manager: Clinton Davis 


\section{CARIBBEAN ISLANDS-Continued}

\section{Martinique}

G Arrondissement Mineralogique de la Guyane

B.P. 458

Fort-de-France

\section{Puerto Rico}

G Mineralogy and Geology Section Industrial Laboratory

Economic Development Administration Box 38, Roosevelt Station

Hato Rey 00929

Head: Carlos Vincente

G Department of Natural Resources Area of Science and Technology Box 5887 Puerta de Tierra Sta. San Juan 00906

C Division of Maps and Topography Department of Public Works

Stop 22

Santurce 00910

$\mathrm{H}$ Aqueduct and Sewer Authority

P.O. Box 7066

Barrio Obrero Station

San Juan 00916

H Water Resources Authority

G.P.O. Box 4267

San Juan 00936

\section{Trinidad and Tobago}

G, R Ministry of Petroleum and Mines P.O. Box 96

Port-of-Spain, Trinidad

C Lands and Surveys Department

Mapping and Control Section

2b, Richmond Street

Port-of-Spain, Trinidad

\section{CENTRAL AMERICA}

\section{Costa Rica}

G Dirección de Geología, Minas y Petróleo

Ministerio de Economía, Industria, y Comercio

Aptdo. 10216

San José

Director: Rolando Castillo M.

\section{CENTRAL AMERICA-Continued}

Costa Rica-Continued

C, G Instituto Geográfico Nacional Aptdo. 2272

San José

Director: Mario Barrantes Ferrero

H National Water Supply and Sewerage Service (SNAA) San José

El Salvador

G Centro de Estudios y Investigaciones Geotécnicas

Ministerio de Obras Públicas

1-A Calle Poniente 925

San Salvador

Director: Mauricio Aquino Herrera

C Instituto Geográfico Nacional

Calle Juan Bertis No. 59

San Salvador

Director: José Alberto Gonzalez Garcia

$\mathrm{H} \quad$ Ministry of Agriculture Irrigation Department San Salvador

\section{Guatemala}

G División de Geología Instituto Geográfico Nacional Avenida las Américas 5-76, Zona 13 Guatemala City

Director: Oscar Daniel Salazar

G, R Dirección General de Mineria e Hidrocarburos

10a Calle 11-46, Zona 1

Guatemala City

Director General: Jorge Godoy Orantes

C Instituto Geográfico Nacional

Avenida las Américas 5-76, Zona 13

Guatemala City

Director: Federico Hernandez Cruz

H División de Recursos de Agua

Instituto Geográfico Nacional Avenida las Américas 5-76, Zona 13 Guatemala City 
CENTRAL AMERICA-Continued

\section{Honduras}

G Dirección General de Minas e Hidrocarburos

Secretaria de Recursos Naturales

Tegucigalpa

C Instituto Geográfico Nacional Barrio La Bolsa, (Comayaguela)

Tegucigalpa

Director General: Fernando Lanza Sandoval

\section{Nicaragua}

G Servicio Geológico Nacional Ministerio de Economía, Industria, y Comercio Aptdo. Postal 1347

Managua, D.N.

Director: Orlando Rodriquez Marenco

C Instituto Geográfico Nacional Ministerio de Fomento y Obras Públicas Aptdo. 2110

Managua, D.N.

Director: Humberto Porta Caldera

C Dirección General de Cartografía

Ministerio de Fomento y Obras Públicas Edificio Cerna, Calle Central Managua, D.N.

\section{Panama}

G Administración de Recursos Minerales

Aptdo. Postal 9658, Zona 4

Panama City

Director: Jorge Quiros

C Instituto Geográfico Nacional "Tommy Guardia"

Aptdo. Postal 5267, Zona 5

Panama City

Director: Julio Mock C.

$\mathrm{H} \quad$ Instituto de Acueductos y Alcantarillados Nacionales

Panama City

Director: Federico G. Guardia
SOUTH AMERICA

\section{Argentina}

G Dirección Nacional de Geología y Minería

Avenida Julio A. Roca 651

Buenos Aires

Director: Edgardo Menoyo

G Servicio Nacional Minero Geológico

Ministerio de Industria y Minería

Buenos Aires

Director: Vincente Hector Padula

G Gerencia de Exploración

Yacimientos Petrolíferos Fiscales

Avenida Rogue Saenz Pena 777

Buenos Aires

C Instituto Geográfico Militar

Av. Cabildo 381

Buenos Aires

Director: Luis María Miró

R Secretaria de Estado de Minería

Avenida Julio A. Roca 651,10 piso

Buenos Aires

Secretary: Luis María Gitelli

\section{Bolivia}

G Servicio Geológico de Bolivia Ministerio de Minas y Petróleo

Avenida 16 de Julio No. 1769

Casilla de Correo 2729

La Páz

Director: José Guillermo Torrez

G Instituto Boliviano del Petróleo

Casilla 4722

La Páz

President: José Patiño

C Instituto Geográfico Militar y

Catastro Nacional

Ave. Saavedra, Miraflores

Commandant: Oscar Wilde Fernandez

$\mathrm{H}$ Oficina de Aguas Potables por

Communidades

La Páz

Chief: P. A. Parada P.

R Ministerio de Minas y Petroleo

Avenida 16 de Julio No. 1769

La Páz

Minister: Ernesto David Pereira 
SOUTH AMERICA-Continued

Brazil

G Companhia de Pesquisa de Recursos Minerais

Avenida Pasteur 404

Praia Vermelha

Rio de Janeiro, GB

President: Yvan Barretto de Carvalho

G Divisão de Geología e Mineralogia

Departamento Nacional de Produção Mineral

Setor de Autarquia Norte

Anexo ao Edifício Petrobras

Brasília, D.F.

Director General: Acyr Avila da Luz

G Instituto de Pesquisas Especiais (INPE)

Caixal Postal 515

São José dos Campos

São Paulo

Director General : Fernando de

Mendonca

C Instituto Brasileiro de Geografia e

Estatistica

Avenida Franklin Roosevelt, 146

Guanabara, Rio de Janeiro

Director: Miguel Alves de Lima

C Directoria do Servico Geografico do

Exercito

Ministerio de Exercito

Rio de Janeiro

H Departamento Nacional de Aguas e

Energía Elétrica (DNAEE)

Ministério das Minas e Energia

Esplanada dos Ministérios

Bloco $\mathrm{J}, 3^{\circ}$ andar

Brasilia, D.F.

Director General: José Duarte de

Magalhães

Chile

G Instituto de Investigaciónes Geológicas

Agustinas 785, Casilla 10465

Santiago

President of the Council: Jorge Leon

Villarreal
SOUTH AMERICA-Continued

Chile-Continued

G Empresa Nacional del Petroleo

Ahumada $341-8^{\circ}$ Piso

Casilla 3556

Santiago

Director: Eduardo Simian G.

G Instituto de Geología

Faculdad de Ciencias Fisicas y

Matematicas

Plaza Ercilla 803

Casilla 13518

Santiago

Director: Cedomir Marangunic D.

G Departamento de Minería

Corporacion de Fomento de la Produccion

Bandera $341-7^{\circ}$ Piso

Jefe: Rene Dintrans A.

C Servicio Aereo Fotogrametrico

Casilla 67

Correolos Cerrillos

Santiago

Comandante: Sergio Piñeiro C.

C Instituto Geográfico Militar

Castro 354

Santiago

Director: José Mutis Puccio

$\mathrm{H}$ Instituto Hidrográfico de la Armada

Errazuriz 232

Casilla 324

Valparaiso

Captain: Raul Herrera A.

\section{Colombia}

G Instituto Nacional de Investigaciones Geológico-Mineras

Carrera 30, No. 48-51

Aptdo. Nacional 2504

Bogotá, D. E.

Director: Alberto Alvarez O.

C Instituto Geográfico "Agustín Codazzi”

Avenida Ciudad de Quito 48-51

Bogotá, D. E.

Director: Alvaro Gonzalez Fletcher 
SOUTH AMERICA-Continued

Ecuador

G Servicio Nacional de Geología y Minería Ministerio de Industrias y Comercio

Casilla 23-A, Avenida 10 de Agosto 666 Quito

Director: Edmundo Lanas C.

C Instituto Geográfico Militar

El Dorado

Quito

Director: Leonardo Endara Romero

H Dirección Nacional de Meteorología y Hidrología

Avenida 10 de Agosto 2627

Quito

Director: Eduardo Mancheno García

R Dirección General de Minas e

Hidrocarburos

Avenida 10 de Agosto 666

Quito

Director General: Gonzalo Sánchez L.

\section{French Guiana}

G Bureau des Recherches Géologiques et Minières

B.P. 42

Cayenne

\section{Guyana}

G Geological Surveys and Mines

Department

P.O. Box 1028

Georgetown

Commissioner of Geology and Mines:

M. A. Lee

C Cartography Section, Lands Division

Ministry of Agriculture and Natural

Resources

Victoria Law Courts

Georgetown

$\mathrm{H} \quad$ Ministry of Works and Hydraulics

Georgetown

\section{Netherlands Antilles}

C Bureau voor Kadaster en Kaartering

Willemstad

Curaçao
SOUTH AMERICA-Continued

\section{Paraguay}

G Dirección de la Producción Mineral Tucarí 271

Asunción

Director: Ricardo Mazo

C Instituto Geográfico Militar

Avenidas Artigas y Vía Ferrea

Asunción

Director General : Abraham Abed

Peru

G Servicio de Geología y Minería

Paz Soldan 225

San Isidro, Aptdo. 889

Lima

Director: Eleodoro Bellido

G Instituto Geofísico del Perú

Aptdo. 3747

Lima

Director: Alberto A. Giesecke M.

C Instituto Geográfico Militar

Avenida Andrés Aramboru 1198

Aptdo. 2038

Lima

Director General: Guillermo Fernandez. Davila Noriega

C Servicio Aerofotográfico Nacional

Base Aérea de las Palmas-Barranco

Lima

Director: Frank Tweddle Granda

H Ministry of Public Health

Department of Sanitary Engineering

Lima

H Dirección de Irrigación

Ministerio de Fomento y Obras Públicas

Lima

R Dirección General de Hidrocarburos

Ministerio de Energía y Minas

Lima

Director: Felix Guerra Chavez

R Oficina Nacional de Evaluación de

Recursos Naturales

Aptdo. 4992

Lima

Director General: Jose Lizarraga R. 
SOUTH AMERICA—Continued

\section{Surinam}

G Geologisch Mijnbouwkundige Dienst

Klein Wasserstraat 1 (2-6)

Paramaribo

Director: G. Doeve

C Centraal Bureau Luchtkartering

P.O. Box 971

Paramaribo

\section{Uruguay}

G Instituto Geológico del Uruguay

Calle J. Herrera y Obes 1239

Montevideo

Director: Mario Gil Nin

G Servicio Oceanográfico y de Pesca

Julio Herrera y Obes 1467

Montevideo

Director: Luis Franzini

C Servicio Geográfico Militar

Avenida 8 de Octubre 3255

Montevideo

Director: Leonardo Pastorino

C Ministerio de Obras Públicas

Dirección de Topografía

Sarandi 90

Montevideo

Venezuela

G, H Dirección de Geología

Ministerio de Minas e Hidrocarburos

Torre Norte, Piso 19

Caracas

Director: Enrique M. Araujo

C Dirección de Cartográfia Nacional

Edificio Camejo, $2^{\circ}$ Piso

Av. Este 6, Centro Simón Bolívar

Caracas

Director: Adolfo C. Romero M.

H Dirección de Recursos Hidraulicos

Ministerio de Obras Publicas

Caracas

Director: José Ramón Velasco Guerra

R Ministerio de Minas y Petróleo

Torre Norte, Piso 20

Caracas

Director: Valentin Hernandez

\section{EUROPE}

Albania

G Ministry of Industry and Mining Tirane

C Association of Scientific Workers of the Peoples Republic of Albania Tirane

H Instituti Hidrometéorologjik Tirane

Austria

G Geologische Bundesanstalt Rasumofskygasse 23

A-1031 Vienna

Director: F. Ronner

C Österreichische Kommission für Internationale Erdmessung Gusshausstrasse 27-29

A-1040 Vienna

President: Friedrich Hauer

C Bundesamtes für Eich- und Vermessungwesen

Krotenthallergasse 3

A-1080 Vienna

H Hydrographisches Zentralbüro

Marxergasse 2

A-1030 Vienna

Secretary: H. Schimpf

R Austrian Supreme Mining Authority Stubenring 1

A-1010 Vienna

Minister: Otto Gassner

R Austrian Oil Administration

Otto Wagnerplatz 5

A-1090 Vienna

Director General: Ludwig Bauer

\section{Belgium}

G Service Géologique de Belgique 13 rue Jenner, Parc Leopold Brussels 4 Inspecteur General : A. Delmer

C Institut Géographique Militaire 13 Abbaye de la Cambre Brussels 4 


\section{EUROPE-Continued}

Belgium-Continued

C Ministère des Travaux Publiques

Service de Topographie et de

Photogrammetrie

155 Rue de la Loi

Brussels 4

$\mathrm{H} \quad$ L'Association Nationale des Services d'Eau (ANSEAU)

Antwerp

President: G. A. Schroeyers

R Ministère des Affaires Économique 23 Square de Meeus

Brussels 4

Minister: Edmond Leburton

R Ministère des Affaires Économique Administration de l'Energie

26 Rue J. A. de Mot

Brussels 4

Director General: J. Martens

Petroleum-Gas: L. de Schrijver

\section{Bulgaria}

G Direktsiià za Geolozhki in Minni Prouchvaniià

Ministère des Mines et des Richesses du Sous-Sol

Sofia

G Geologicheski Institut

Būlgarska Akademina na Naukite

"7 Nomeuri" 1

Sofia

Deputy Director: B. Bogdanov

C Research Institute of Geodesy and Cartography

Sofia-Pavlova, Musala 1

Director: D. Stojčev

H Research Institute for Hydrology and Meteorology

Academy of Sciences

Blvd. Lenin No. 66

Sofia

\section{EUROPE-Continued}

\section{Czechoslovakia}

G Ustav Geologický

[Geological Institute]

Ceskoslovenská Akademie ved

[Czechoslovak Academy of Sciences]

Lysolaje 6

Pošta Suchodol 2

Praha 6

G Ústředni Ústav Geologicky

Malostranské námèstí 19

Praha 01

Director: J. Svoboda

C Vojenský Zeměpisný Ústav

Vyznamenaný Řáděm Rudé Hvezdy

Praha 6

H Hydrometeorologický Ústav

[Surface and Ground Water Resources]

Holečkova 8

Praha 5

Director: J. Zitek

Denmark

G Danmarks Geologiske Undersøgelse

31 Thoravej

DK 2400 København

Director: Ole Berthelsen

C Geodetic Institute

Rigsdagsgaarden 7

DK 1218 København

Director: Einar Andersen

R Handelsministeriet

[Ministry of Commerce]

Beredskabskontoret

12 Slotholmsgade

DK 1216 København

\section{Finland}

G Geologinen Tutkimuslaitos

02150 Otaniemi

Director: Herman Stigzelius

C Geodeettinen Laitos

[Geodetic Institute]

Pasilankatu 43A

00240 Helsinki

Director: T. J. Kukkamaki 
EUROPE-Continued

Finland-Continued

C Land Survey Board

Kirkokkatu 3

00170 Helsinki

Director: Kantee

$\mathrm{H}$ National Board of Public Roads and

Waterways Construction

Et. Esplanadikatu 4

00130 Helsinki

Director : Niskala

\section{France}

G Centre National de la Recherche

Scientifique

15 Quai Anatole France

75700 Paris

G Institut Français du Petrol, de

Condurontes, et Lubriesiants

4 Avenue de Bois-Preau

92502 Rueil Malmaison

Director: J. C. Balaceanu

G Bureau des Recherches Géologiques et Minières (BRGM)

B.P. 6009

45108 Orléans-La Source 02

Director: Claude Beaumont

G, H Office de la Recherche Scientifique et

Technique Outre-Mer (ORSTOM)

[Office of Scientific and Technical Research Overseas]

24 rue Bayard

75008 Paris

President: A. Valabregue

C Institut Géographique National

136 bis Rue de Grenelles

75700 Paris

Director: Georges Laclavere

C Service de Documentation et de Cartographie Géographiques

191 rue Saint Jacques

75800 Paris

Director: J. Dresch

H Electricité de France

134 Boulevard Hussmann

75336 Paris Dex 08

\section{EUROPE-Continued}

Federal Republic of Germany (West)

G Geological Institut

TU Clausthal

3392 Clausthal-Zellerfeld

Bonn

G Bundesanstalt für Geowissenschaften und Rohstoffe (BGR)

Alfred-Bentz-Haus

Postfach 510153

Stilleweg 2

3 Hannover 51

Acting President: F. N. Bender

C Institut für Angewandte Geodasie

Kennedyallee 151

Frankfurt am Main

C Arbeitsgemeinshaft der Vermessunguerwaltungen der Länder der Bundesrepublik Deutschland (AdV)

Warmbüchenkamp 2

Hannover

R Oberbergamt Clausthal-Zellerfeld

[Mining authority]

3392 Clausthal-Zellerfeld

Hindenburghplatz 9

Bonn

Director: Rudolf Koch

German Democratic Republic (East)

G Zentrales Geologisches Institut

104 Berlin Invalidenstrasse

Berlin

Director : Karl Schmidt

\section{Great Britain}

G Institute of Oceanographic Sciences

Wormley, Godalming

Surrey GU8 5UB

Director: H. Charnock

G Directorate of Overseas Surveys

Foreign and Commonwealth Office

Kingston Road

Tolworth, Surbiton

Surrey KT5 9NS

Director: D. E. Warren 


\section{EUROPE-Continued}

\section{Great Britain-Continued}

G Institute of Geological Sciences

Exhibition Road, South Kensington

London, SW7 2DE

Director: Kingsley Dunham

C Directorate of Military Survey

Ministry of Defense

Elmwood Avenue

Feltham, Middlesex

Director: J. Kelsey

C Ordinance Survey of Great Britain

Ramsey Road, Maybush

Southhampton SO9 4DH

Director: B. St. G. Irwin

$\mathrm{H} \quad$ Institute of Hydrology

Natural Environment Research Council

Howvery Park, Wallingford

Berkshire

Director: J. S. G. McCulloch

\section{Greece}

G, H Institouton Geologias kai Ereynon Ypedaphous

[Institute of Geologic and Subsurface

Research]

Amerikis No. 6

Athens

C Hellenic Army Geographical Service

Athens

R National Petroleum Service

Ministry of Coordination

4 Karageorgi Servias Street

Athens 125

Director General: N. Mavrakis

\section{Hungary}

G Magyar Allami Földtani Intézet

[Hungarian State Geological Institute]

Népstadion-ut 14, Pf. 106

Budapest 1442

Director: Jozsef Konda

G Magyar Allami Eötvös Loránd Geofizikai Intézet

[Hungarian Geophysical Institute]

Kolombusz p. 17-23

Budapest XIV

Director: Pál Müller

\section{EUROPE-Continued}

Hungary-Continued

G Magyar Tudományos Akadémia Geokémiai Kutató Laboratóriuma [Geochemical Research Laboratory]

Museum-krt. 4/a

Budapest 1088

Director: Elemér Szádeczky-Kardoss

G, C Magyar Tudomános Akadémia

Geodéziai és Geofizikai Kutató Intézete

[Geodetic and Geophysical Research]

Múzeum u. 6

Sopron 9400

Director: Jozsef Somogyi

C M. B. M. Országos Foldúgyi és Térképészeti Hivatal

[National Cadaster and Cartography Bureau]

Kossuth Lajos tér 11

Budapest V

H Vizgazdálkodási Tudományos Kutató Intézet

[Research Institute for Water Resources Development]

Rákóczi út 41

Budapest 1088

Director: Károly Stelczer

\section{Iceland}

G Natural Heat Section

National Energy Authority

Reykjavik

Head: Gudmundur Palmason

Chief Geologist: Jon Jonsson

C Icelandic Survey Department

Laugavegi 178

Reykjavik

\section{Ireland}

G Geological Survey of Ireland

14 Hume Street

Dublin 2

C Ordinance Survey of Ireland

Phoenix Park

Dublin 


\section{EUROPE-Continued}

Ireland-Continued

R Establishment and Fuel and Power Division

Department of Transport and Power

Kildare Street

Dublin 2

Principal Officer: D. S. O’Maolain

Italy

G Istituto Internazionals di Vulcanologia

Viale Regina Margherita 6

Catania

Director: G. Marinelli

G Servizio Geologico d'Italia

Ministero dell'Industria del Commercio e dell'Artigianato

Direzione Generale della Miniere

Largo di Santa Susanna 13

Roma

Director: Alfredo Iacobacci

C Instituto Geografico Militare

Via Cesare Battisti 10

Firenze

R Ministero Industria e Commercio e Artigianato

Direzione Generale dell'Industrie di

Base e delle Fonti di Energia

Via Molise 43

Roma

\section{Liechtenstein}

G Geological Survey of Liechtenstein Vaduz

\section{Luxembourg}

G Service Géologique de Luxembourg

Direction des Ponts et Chaussées

13, rue J. P. Koenig

Luxembourg

Director: Michel Lucius

C Administration du Cadastre et de la Topographie

54 Avenue Gaston Diderich

Luxembourg

Malta

C Department of Information

24 Merchants Street

Valletta

\section{EUROPE-Continued}

\section{Netherlands}

G Rijks Geologische Dienst

Spaarne 17, P.O. Box 157

Haarlem

Director: A. A. Thiadens

C Topografische Dienst

Westvest 9

Delft

H Ministry of Transport and Waterways

Bureau of Water Control and Works

The Hague

Director: A. Volker

R Ministry of Economic Affairs

Directorate General for Energy

Bezuidenhoutsweg 30

The Hague

Minister : L. de Block

\section{Northern Ireland}

G Geological Survey of Northern Ireland 20 College Gardens

Belfast

\section{Norway}

G Geofysiske Kommisjon

Blindern

Oslo 3

President: R. Fjørtoft

G, C Norsk Polarinstitutt

Rolfstangveien 12,

Postboks 158

Oslo Lufthavn

Director: Tore Gjelsvik

G Norges Geologiske Unders $\varnothing$ kelse

P.B. 3006 Ostmarkneset

Leiv Erikssons Vei 39

Trondheim

Managing Director: Knut S. Hejer

C Norges Geografiske Oppmaling

St. Olavs Gt. 32

Oslo

H Norwegian Institute for Water Resources

Council for Science and Industry

Gaustadalleen 25

Blindern

Oslo 
EUROPE-Continued

Norway-Continued

H Water Resources and Electricity Board Water Resources Division

P.O. Box 5091

Oslo

Chief: A. H. Hjelm-Hansen

R Norwegian Petroleum Directorate

Lagaards Veien 80,

Stavanger

Director : Fredrik Hagemann

Poland

G Zaklad Paleozoologii

Zwirki i Wigury

Warsaw

Director: Zofia Kielan-Jaworowska

G Instytut Geophysica

Pasteura 3

Warsaw

Director: Roman Tiesseyre

G Glówny Instytut Górnictwa

[Central Mining Institute]

Plac Gwarków I

Katowice

Director: Erast Konstantynowicz

G Centrainig Urzad Geologii

[Research Center for Geological

Sciences]

Zwirki i Wigury 93

Warsaw

Director: M. Książkiewicz

G, H Instytut Geologiczny

ul. Rakowiecka 4

Warsaw

Director: Roman Osika

G Instytut Naftowy

[Institute of Petroleum]

ul. Lubicz 25a

Cracow

Director: Jósef Gumuxczyḿski

C Centralny Urzad Geodezji i Kartografii Warsaw
EUROPE-Continued

Poland-Continued

H Instytut Meteorologii i Gospodarki Wodnej

Podleśna

Warsaw

Director: Eryk Bobiński

Portugal

G Direcção Geral de Minas e Servicos Geológicos

Ministério da Economia

Rua António Enes, 5

Lisbon

Director: Fernando Soares Carneiro

G Serviços Geológicos de Portugal

Rua da Academia das Ciências 19

Lisbon 2

Director: Fernando Moitinho de Almeida

C Instituto Geográfico e Cadastral

Ministério das Finanças

Praça da Estrels

Lisbon

H Estação Áqüícola

[Water Research Station]

Villa do Conde

Lisbon

Director: Joaquim Antonio Soares Soeiro

R Secretary of State for Industry

Avenida Miguel Bombarda, 6

Lisbon 1

Director: Francisco Goncalves

Cavaleiro de Ferreia

\section{Romania}

G Institutul de Geofizicà Aplicatà

Str. Izvor 78

Bucharest 5

Director: D. Romanescu

G Institutul de Geologie şi Paleontologie

Universitatea "Babes-Bolyai"

Str. M. Kogalniceanu 1

Cluj

Director: O. Protescu 


\section{EUROPE-Continued}

\section{Romania-Continued}

G Institutul Geologie

Sos Kiseleff $\mathbf{5 5}$

Bucharest

Director: Marcian Bleahu

C Institutul de Geologie şi Geografie

Str. Dr. Burghele 1

Bucharest

H Institutul de Meteorologie şi Hidrologie

Sos Bucuresti Ploieşti 97

Bucharest

Director: N. Ciovică

R Ministry of Mining, Petroleum, and Geology

Str. Mendeleev 36-38

Bucharest

Minister: Bujor Amasan

R Ministry of Petroleum

Blvd. Republicii 32

Bucharest

Spain

G Instituto Nacional de Geofísica

Serrano 123

Madrid

Director : Luis Lozano Calvo

G Servicio de Geología de Obras Publicas 81 Avenida de Portugal

Madrid

Director: Manuel Gomez de Pablos

G Empresa Nacional ADARO de Investigaciones Mineras, S. A.

Serrano, 116

Madrid

Director: Juan Antonio Gómez-Angulo

G Instituto Geológico y Minero de España

Rios Rosas 23

Madrid

C Instituto Geográfico y Catastral

Calle del General Ibáñez de Ibero 3

Madrid

Director General: Juan García

Frías y García
EUROPE-Continued

Spain-Continued

$\mathrm{H} \quad$ Instituto de Hidrología

Patronato "Alfonso el Sabio"

Paseo Bajo de la Virgen del Puerto 3

Madrid

President (of Patronato) :

P. Antonio Romaná

R Ministerio de Industrias

Serrano 35

Madrid

Minister: Alfredo Santos Blanco

Sweden

G, H Sveriges Geologiske Undersokning S10405 Stockholm

Director: Leif Andreasson

G Norrlandsfonden

[Northland Foundation]

Smedjegatan 17

S95100 Lulea

Chairman: Gunnar Lange

G Statens Geotekniska Institut

(Engineering Geology)

Banergatan 16

S11526 Stockholm

Director: Bengt B. Broms

C Rikets Allmanna Kartverk

[Geographical Survey Office]

Fack

S16210 Vällingby

Director: Harry Wikström

$\mathrm{H} \quad$ Sveriges Meteorological och Hydrologiska Institut

Fridhensgatan 9

P.O. Box 12108

10223 Stockholm

Director: Alf Nyberg

\section{Switzerland}

G, H Schweizerische Geologische Kommission Naturforschende Gesellschaft

4000 Bernoultistr. 32

Basel

Director: A. Spicher 


\section{EUROPE-Continued}

\section{Switzerland-Continued}

C Bidgenossische Landestopographie [Topographical Survey of Switzerland] Seftigenstrasse 264 Director : E. Huber

H Office Fédéral de l'Economie Hydraulique 3011 Berne

H Service Hydrographique

Bollwerk 27

Berne

R Eidgenossisches Amt Fuer Energiewirtschaft

Erdoel und Gas Wirtschaft

Kapellenstrasse 14

3011 Berne

Director: Hans Rudolf Siegrist

Union of Soviet Socialist Republics (USSR)

G All-Union Geological Scientific Research Institute (VSEGEI)

Sredny Prospekt 72B

Leningrad 199026

Director: A. Zhamoida

G Geologic Institute

Akademiya Nauk SSSR

Pyzhevsky per. 7,

Moscow G-17

Director: A. V. Peyve

G Vernadsky Institute of Geochemistry

Akademiya Nauk SSSR

Vorobievskoe schosse 47 a

Moscow

Director: A. P. Vinogradov

G Institute of Earth Physics

Akademiya Nauk SSSR

B Gruzinskay ul., 10

Moscow D-242,

Director: M. A. Sadovsky

G Section of Earth Sciences

Department of Geology, Geophysics, and Geochemistry

Akademiya Nauk SSSR

Leninsky Prospekt 14

Moscow

Academician-Secretary (of

Department) : V. I. Smirnov

\section{EUROPE-Continued}

USSR_Continued

G Institute of Oceanology

Department of Oceanology, Atmospheric Physics, and Geography

Letnaya ul 1,

Lyublino

Director: A. S. Monin

G Institute of Volcanology

Siberian Department

Akademiya Nauk

Porgranichaya ul. 3

Petropavlovsk, Kamchatka

Director: G. S. Gorshkov

G Institute of Geology and Geophysics

Siberian Department

Akademiya Nauk

Akademgorodok

Novosibirsk

Director: A. A. Trofimuk

G Institute of Frozen Soils

Siberian Department

Akademiya Nauk

Yakutsk

Director: P. I. Melnikov

G Institute of the Earth's Crust

Siberian Department

Akademiya Nauk

ul. Lermontova 128

Irkutsk

Director: M. M. Odintsov

G Ministry of Geology of the USSR

Bolshaya Gruzinskaya, 416

Moscow D-242

C Glavnoe Upravlenie Geodezzii

I Kartografii

[Main Administration for Geodesy and Cartography]

Ministerstva Vnutrennikh del SSSR

Moscow

Deputy Director: L. A. Kashin

H Hydrometeorological Service

12, Pavlik Morozov Street

Moscow D-376 
EUROPE-Continued

USSR_Continued

$\mathrm{H} \quad$ Institute of Hydrodynamics

Siberian Department

Akademiya Nauk

Akademgorodok

Novosibirsk

Director: M. A. Lavrentiev

\section{Yugoslavia}

G Zavod za Geološki i Geofizička Istraživanja

[Institute for Geological and

Geophysical Research]

Post-fah 227

Kamenicka 16

Belgrade

Director: Živojin Djordjević

G Geoinženjering Sarājevo

Institut za Geotehnikui Hidrogeologiju,

Ilidza

Director: Safet Cicic

G Institute for Geological and Mining Exploration and Investigation of Nuclear and other Mineral Raw

Materials

Rovinjska 12

Belgrade

Director General: Milorad Nikolic

C Glavna Geodetska Uprava Pri Vladi Federativne

Narodne Republike Jugoslavije

Belgrade

$\mathrm{H}$ Institute for Development of Water Resources

Vojvo de Mišića 43

Belgrade

H Federal Hydrometeorologica Institute

Bercaninova 6

Belgrade

R Nafta-Poslovno udruzenje

Marticeva 65

Zagreb

\section{AFRICA}

Algeria

G Service Géologique de l'Algérie

Immeuble Mauretania-Agha

Boulevard Colonel Amirouche

Agha

Director: Omar Merabet
AFRICA-Continued

Algeria-Continued

C Institut National de Cartographie

Ministère de la Defense Nationale

B.P. 32

Hussein Dey

C Institut Géographique National

20 rue Abane Ramdane

Algiers

Director: M. Pinson

H Hydrology Department

Ministry of Public Labor

Algiers

Director: Mohammed Benblidia

R Ministère de l'Industrie et de l'Ènergie

Immeuble le Colisée

Rue Zephirin Roccas

Algiers

Minister: Belaid Abdesslam

Angola

G, C, Direcção des Serviços de Geología e Minas

R Caixa Postal 1260-C

Luanda

Director: Jorge Manuel Trigo de Mira

Botswana

G, C Geological Survey and Mines

Department

P.O. Box 94

Lobatsi

Director: J. V. Hepworth

$\mathrm{H}$ Department of Water Affairs

Ministry of Commerce, Industry, and

Water Affairs

P.B. 29

Gaborone

Permanent Secretary: P. W. Reardon

\section{Burundi}

G Ministère des Affaires Economiques et Financières

Départment de Géologie et Mines

B.P. 745

Bujumbura

Director: Raphael Banciyeko

\section{Cameroon}

G Direction des Mines et de la Géologie du

Cameroun

B.P. 70

Yaoundé 


\section{AFRICA-Continued}

Cameroon-Continued

G Office de la Recherche Scientifique et

Technique Outre-mer Centre

ORSTOM de Yaoundé ${ }^{1}$

B.P. 193

Yaoundé

Director: R. Lefevre

C Institut Géographique National

B.P. 157

Yaoundé

Director: J. Larivé

\section{Central African Republic}

G, C Ministère des Travaux Publiques, des Transports, des Communications et des Mines

B.P. 737

Bangui

Director: M. Dubois

G, R Office de la Recherche Scientifique et Technique Outre-mer Centre ORSTOM de Bangui ${ }^{1}$

P.B. 893

Director: Y. Chatelin

\section{Chad}

G, C Direction des Mines et de la Géologie

B.P. 816

Fort-Lamy

Director: Omar Abdoul

G Bureau de Recherches Géologiques et Minières ${ }^{1}$

B.P. 449

Fort-Lamy

Director: M. Abadie

G Office de la Recherche Scientifique et Technique Outre-mer Centre ORSTOM de Fort-Lamy ${ }^{1}$

B.P. 65

Fort-Lamy

Director: P. Audry

Congo, Republic of

G Service des Mines et de la Géologie

B.P. 12

Brazzaville
AFRICA-Continued

\section{Congo, Republic of-Continued}

G Bureau de Recherches Géologiques et Minières ${ }^{1}$

B.P. 431

Brazzaville

Director: M. Nicault

C Institut Géographique National

Centre en Afrique Equatoriale

P.B. 125

Brazzaville

\section{Dahomey}

G Direction des Mines, de la Géologie, et des Hydrocarbures

Ministère des Travaux Publiques, des Transports et des Telecommunications

B.P. 249

Cotonou

Chief : Julien C. Laleye

G, H Office de la Recherche Scientifique et

Technique Outre-mer Centre ORSTOM de Cotonou ${ }^{1}$

B.P. 390

Cotonou

Director: P. Viennot

C Service Topographique

Ministère des Travaux Publiques

B.P. 360

Cotonou

Ethiopia

G Geological Survey

Ministry of Mines

P.O. Box 486

Addis Ababa

C Government Mapping and Geography Institute

P.O. Box 597

Addis Ababa

Director: D. Assaye

$\mathrm{H} \quad$ Water Resources Department

Ministry of Public Works and Communication

P.O. Box 1008

Addis Ababa

\footnotetext{
${ }^{1}$ Designates French research organization, without governmental affiliation.
} 
AFRICA-Continued

Ethiopia-Continued

R Ministry of Mines

P.O. Box 486

Addis Ababa

Minister: Ato Emmanull Abraham

French Territory of Afars and Issas

G Services des Travaux Publiques

Djibouti

\section{Gabon}

G, C Bureau de Recherches Géologiques et Minières

B.P. 175

Libreville

Director: M. Arnould

$\mathrm{H} \quad$ Office de la Recherche Scientifique et Technique Outre-mer, Centre ORSTOM de Libreville ${ }^{1}$

B.P. 3115

Libreville

Director: D. Martin

R Direction des Mines

B.P. 576

Libreville

\section{Gambia}

C Survey Department

Bathhurst

\section{Ghana}

G Geological Survey of Ghana

P.O. Box M 80

Ministry Branch Post Office

Accra

C Ghana Geographical Association

University of Ghana

Legon

President: E. A. Boateng

C Survey Division

P.O. Box 191

Cantonments

Accra

Chief Survey Officer: R. J. Simpson

AFRICA-Continued

Ghana-Continued

H Water Resources Research Unit

Council for Scientific and Industrial

Research

P.O. Box M 32

Acera

Officer in charge: E. Lartey

\section{Guinea}

G Services des Mines et de la Géologie Conakry

\section{Ivory Coast}

G Service Géologique

Direction de la Géologie et de la Prospection Minière

Ministère de l'Economie et des Finances B.P. 1368

Abidjan

Director: Paul Ahui

C Ministère des Travaux Publiques et des Transports

B.P. 20952

Abidjan

G, C, Société pour le Developpment Minier de la Côte d'Ivoire (SODEMI)

B.P. 2816

Abidjan

\section{Kenya}

G Geological Survey of Kenya

Mines and Geological Department

Ministry of Natural Resources

P.O. Box 3009

Nairobi

Director: B. N. Baher

C Survey of Kenya

P.O. Box 30046

Nairobi

Director: P. P. Anyumba

$\mathrm{H} \quad$ Water Department

Ministry of Agriculture

P.O. Box 30521

Nairobi

\footnotetext{
IDesignates French research organization, without governmental
} 


\section{AFRICA-Continued}

\section{Lesotho}

G Geological Survey Department Department of Mines and Geology P.O. Box 750

Maseru

Head: W. C. Fairbain

C Township Survey Department P.O. Box 20 Maseru

H Hydrology Department

Maseru

Director: Thomas R. Hyde

\section{Liberia}

G, H Liberian Geological Survey Ministry of Lands and Mines

P.O. Box 9024

Monrovia

Director: Cletus S. Wotorson

C Liberian Cartographic Service

Ministry of Lands and Mines

P.O. Box 9024

Monrovia

Director: J. L. F. Sawyerr

G, C, Ministry of Lands and Mines

H, R P.O. Box 9024

Monrovia

Minister: A. E. Nyema Jones

Libya

G Geological Research and Mining Department

Industrial Research Center

P.O. Box 3633

Tripoli

Director: Faisal Zaied Ali

C Ministry of Planning and Development

Director of Surveying and Mapping

Bengasi

Director: Muftah Unis

R Ministry of Petroleum Affairs Tripoli

Minister: Khaliifah Muusa

\section{AFRICA-Continued}

Malagasy Republic

G Centre Océanographique

Office de la Recherche Scientifique et

Technique Outre-mer (ORSTOM) ${ }^{1}$

B.P. 68

Nossi-Bé

Director: A. Crosnier

G Service Géologique

Ministère des Mines et de l'Energie

B.P. 280, Armandrianomby

Tananarive

C Institut Géographique National

3 rue Jean Laborde

B.P. 456, Andohalo

Tananarive

Director: M. Perreau-Saussine

H Hydrological Survey

Tananarive

Malawi

G Geological Survey Department

Ministry of Natural Resources

P.O. Box 27, Liwonde Road

Zomba

Director: J. H. Bean

C Survey Department

Ministry of Natural Resources

Box 349

Blantyre

Mali

G Direction National des Mines et de la Géologie

Koulouba, Bamako

C Institut National de Topographie

B.P. 240

Bamako

Director: Bamory Sanogo

H Service Hydrologique

B.P. 66

Bamako

1 Designates French research organization, without governmental affiliation. 


\section{AFRICA-Continued}

\section{Mauritania}

G Direction des Mines et de la Géologie

Ministère de l'Industrialisation et des Mines

B.P. 199

Nouakchott

Director: Y. Barbier

C Service Topographique

B.P. 237

Nouakchott

Director: Habib Ould Ely

\section{Mauritius}

G Ministry of Agriculture and Natural Resources and Cooperative Development

Port Louis

C Ministry of Housing, Lands, and Town and Country Planning

Survey Department

Port Louis

Director: A. P. Rault

\section{Morocco}

G Institut Scientific Chérifien

(Geology, Seismology, Geomagnetism)

Ave. Moulay-Chérif

Rabat

Director: H. Msougar

G Division de la Géologie

Ministère du Commerce, de l'Industrie, des Mines, et de la Marine marchande

Rabat

Director: Moussa Saadi

C Service Topographique

Ministère de l'Agriculture

Avenue Moulay Youssef

Rabat

Director: M. Naceur

$\mathrm{H}$ Direction de l'Hydraulique

Division de Resources en Eau

B.P. 525 Rabat-Chellah

AFRICA-Continued

Morocco-Continued

R Ministère de la Production Industrielle et des Mines

Avenue Mohammed V

Quartier Administratif

Rabat

Under-Secretary of State: Adelaziz Benjelloun

\section{Mozambique}

G Direcçấo dos Serviços de Geologia e Minas

Caixa Postal 217

Lourenço Marques

Director: J. M. Trigo Mira

C Secretary for Lands and Rural Development

Geographical and Cadastral Service

Lourenço Marques

Niger

G, H Service des Mines et de la Géologie

Ministère des Travaux Publiques et des Mines

B.P. 257

Niamey

Director: M. O. Diallo Oumar

C Service Topographique et du Cadastre Ministère des Finances

B.P. Box 250

Niamey

\section{Nigeria}

G, H Geological Survey Division

Ministry of Mines and Power

P.M.B. 20007

Kaduna South

Director: C. N. Okezie

C Land and Surveys Division

Ministry of Works and Housing

Lagos

R Federal Ministry of Mines and Power

Yakubu Gowon Street

Lagos

Commissioner: Shettima Ali Monguno 


\section{AFRICA-Continued}

\section{Nigeria-Continued}

R Federal Ministry of Mines and Power

Broad Street Office

Lagos

Chief Petroleum Engineer: M. O. Feyide

Portuguese Guinea

G Serviços de Geologia e Minas

Caixa Postal 399

Bissau

\section{Reunion}

G Services des Travaux Publiques

St. Denis

\section{Rhodesia}

G Geological Survey of Rhodesia

Ministry of Mines and Lands

P.O. Box 8039, Causeway

Salisbury

Director: F. L. Amn

C Department of Trigonometrical and Topographical Surveys

Salisbury

\section{Rwanda}

G Service Géologique du Rwanda

Ministère du Commerce, des Mines et de l'Industrie

B.P. 15

Ruhengeria

Director: P. Corminboeuf

C, $\mathrm{H}$ Service des Terres

Ministère de l'Agriculture et de

l'Elevage

B.P. 621

Kigali

\section{Senegal}

G Centre de Géophysique

Office de la Recherche Scientifique et Technique Outer-mer (ORSTOM) ${ }^{1}$

B.P. 50, M'Bour

Director: C. Blot

1 Designates French research organization, without governmental affiliation.
AFRICA-Continued

\section{Senegal-Continued}

G Bureau de Recherches Géologiques et Minières

B.P. 268

Dakar

Director: M. Delafosse

C Institut Géographique National

Dakar

$\mathrm{H}$ Direction Energie et Hydraulique

B.P. 4021

Dakar

\section{Sierra Leone}

G Geological Survey Division

Ministry of Lands, Mines, and Labor

New England

Freetown

Director: A. H. Gabiri

C Surveys and Land Division

Ministry of Lands, Mines, and Labor

Freetown

\section{Somalia}

G Geological Survey Department

Ministry of Mining

P.O. Box 744

Mogadiscio

Director: V. N. Kozerenko

C Survey and Mapping Department

P.O. Box 24

Mogadiscio

Director: Musa Adan Wadadid

\section{South Africa}

G, H Geological Survey of South Africa

Department of Mines

P.O. Box 112

Pretoria

Director: J. F. Enslin

G Magnetic Observatory

(Geomagnetism, Seismology)

P.O. Box 32

Hermanus

Head: A. M. van Wijk 
AFRICA-Continued

South Africa-Continued

C Trigonometrical Survey

Rhodes Avenue

Mowbray, Cape Town

Director: F. W. Marsh

H National Institute for Water Research (CSIR)

P.O. Box 395

Pretoria

Director: G. J. Stander

R South African Department of Mines

Oranje-Nassau Building

Schoeman Street

Pretoria

Minister: C. de Wet

R South African Department of Industries Forum Building

Bosman and Struben Streets

Pretoria

Secretary: J. J. Kitsoff

\section{South-West Africa (Namibia)}

G Geological Survey

P.O. Box 2168

Windhoek

C The Surveyor General

Windhoek

Spanish Sahara

G Dirección General de Plazas y Provincias Africanas

Servicio Minero y Geológico

Castellana No. 5

Madrid, Spain

\section{Sudan}

G Geological Survey Department

Ministry of Mining and Industry

P.O. Box 410

Khartoum

Director: Abdel Latif Widatalla

C Topographical Survey Division

Survey Department.

Khartoum

Director: Abdel Wahab Ahmed Hassan

\section{AFRICA-Continued}

Sudan-Continued

H Rural Water and Development Corp.

Chief Engineer's Office

Khartoum

\section{Swaziland}

G, R Geological Survey and Mines Department

P.O. Box 9

Mbabane

Director: D. N. Davis

C Public Works Department

Ministry of Works, Power, and

Communications

P.O. Box 58

Mbabane

Tanzania

G Geological Survey

Mineral Resources Division

Ministry of Commerce and Industries

P.O. Box 903

Dodoma

Director: Alex C. M. McKinley

C Survey Division

Ministry of Lands, Settlement, and Water Development

Dar es Salaam

Director: William Dickson

Togo

G Direction des Mines et de la Géologie

Ministère des Travaux Publiques, Mines, Transports, et Télécommunications

B.P. 356

Lomé

Director: Otto Gartner

C Service Topographique

Ministère des Finances et de l'Economie

Lomé

H Office de la Recherche Scientifique et Technique Outre-mer Centre ORSTOM de Lomé ${ }^{1}$

B.P. 375

Lomé

Director: A. Le Cocq

1 Designates French research organization, without governmental affiliation. 


\section{AFRICA-Continued}

Tunisia

G Service Géologique de Tunisie

95 Avenue Mohamed V

Director: H. Besbes

C Division Topographie

Secretariat d'Etat aux Travaux

Publiques et à l'Habitat

Rue de Jordanie

Tunis

Director: S. Benghachane

H Division des Resources en Eau

41 Rue de la Manoubia

Tunis

H Service de l'Hydraulique et de

l'Equipement rural

Sous-Secrétariat de l'Agriculture

Tunis

R Department des Mines et de l'Energie

Sous-Secrétariat d'État à l'Industrie et au Commerce

Tunis

Chief: Mekki Zidi

R Sous-Secrétariat d'État au Plan à

l'Industrie et au Commerce

Tunis

Chief: Abderrazak Rassa

\section{Uganda}

G Geological Survey and Mines Department

P.O. Box 9

Entebbe

Commissioner: C. E. Tamale-Ssali

C Office of the Commissioner of Lands and Surveys

P.O. Box 7061

Kampala

Commissioner: S. Labeca Okec

$\mathrm{H} \quad$ Ministry of Minerals and Water Resources

Water Development Department

Kampala

\section{United Arab Republic}

G Geological Survey and Mining Authority

Ministry of Industry

Abbasia Post Office

Cairo

Chairman: Rhusdi Said

\section{AFRICA-Continued}

United Arab Republic-Continued

C Survey Department

20 Bagdad Street, El Giza

Cairo

Director: Ibrahim Mostafa Ganem

G, H Academy of Scientific Research and Technology

101 Kasr El Aini St.

Cairo

Director: E. M. El Shazly

H Hydrological Research Station

Kanater-al-Khaiyria Barrages

Cairo

Director: A. A. El-Darwish

R General Petroleum Co.

Dr. Mustafa Abu Zahra Street

Nasr City

Cairo

Head: Ahmed El-Barkouki

\section{Upper Volta}

G Direction de la Géologie et des Mines

B.P. 601

Ouagadougou

Director: Philippe Ouedraogo

C Service Topographique

Ministère des Travaux Publiques

Ouagadougou

H Office de la Recherche Scientifique et Technique Outre-mer Centre ORSTOM a Ouagadougou ${ }^{1}$

B.P. 189

Ouagadougou

Director: H. Barral

Zaire

G Service Géologique du Zaire

B.P. 898

44 Avenue des Huileries

Kinshasa

Director: Gabriel Dembe

C Institut Géographique

106 Blvd du 30 Juin

B.P. 1873

Kinshasa

Director: M. E. Nzungo

1 Designates French research organization, without governmental affiliation. 


\section{AFRICA-Continued}

\section{Zambia}

G Geological Survey of Zambia

Ministry of Mines and Mining

Development

P.O. Box RW 135

Ridgeway, Lusaka

Director: A. R. Drysdall

C Department of Surveys

Ministry of Lands and Natural Resources

P.O. Box RW 397

Ridgeway, Lusaka

Surveyor General: J. L. Seftel

\section{NEAR EAST-SOUTH ASIA}

\section{Afghanistan}

C Department of Mines and Geology

Ministry of Mines and Industries

Darulaman

Kabul

Director: Abdullah Samad Salah

C Institute of Cartography

Ministry of Mines and Industries

Kabul

Director: Muzaffarud Din Yaqubi

$\mathrm{H} \quad$ Water and Soil Survey Department Ministry of Agriculture and Irrigation Kabul

President: Joma Mohd. Mohammadi

\section{Bangladesh}

G Geological Survey

Pioneer Road

Segun Bagicha

Dacca

Director General : Mesbahuddin Ahmed

\section{Cyprus}

G Geological Survey Department

Ministry of Agriculture and Natural

Resources

Nicosia

Director: Yiangos Cos Hj Stavrinou

C Department of Lands and Surveys

Ministry of the Interior

Nicosia

Deputy Minister: Andreas Christofi
NEAR EAST-SOUTH ASIA-Continued

\section{Cyprus-Continued}

H Water Development Department

Ministry of Agriculture and Natural

Resources

Nicosia

Director: Christos Antoniou Konteatis

India

G Central Seismological Observatory Shillong

G Geological Survey of India 27 Jawaharlal Nehru Road

Calcutta 13

Director General: C. Karunakaran

G National Geophysical Research Institute Hyderabad 7

Director: Hari Narain

G Mining, Geological, and Metallurgical Institute

29 Jawaharlal Nehru Road

Calcutta 16

President: K. Z. George

G National Institute of Oceanography

Miramar, Panaji

Goa

Director: N. K. Panikkav

C Geodetic and Geophysical Surveys

Survey of India

Dehra Dun

Director: J. Chatterjee

C Survey of India

Surveyor General Office

P.O. Box 37

Dehra Dun (H.P.)

$\mathrm{H}$ Central Ground Water Board

Faridabad, Haryana

Chairman: B. B. Vorha

R Ministry of Petroleum and Chemicals

Government of India

Dr. Rajendra Prasad Road

New Delhi 1

Minister : Triguna Sen 
NEAR EAST-SOUTH ASIA-Continued

Iran

G Geological Survey of Iran

P.O. Box 1964

Tehrān

Managing Director: R. Assafi

C National Cartographic Centre

P.O. Box 1844, Mahrabad

Tehrān

Director: G. Basseri

C National Geographic Organization

Imperial Iranian Army

Tehrān

$\mathrm{H} \quad$ Institute of Hydro-Sciences and Water Resources Technology

64 Ghadessi Street, North

Boulevard Elizabeth

Tehrān

Director: M. Mozayeny

$\mathrm{H} \quad$ Ministry of Water and Power

Tehrān

Minister: Mansour Rohani

Iraq

G Petroleum Research Institute

Shalijiah

Baghdad

Director: Abood Al-Khafaji

G Geological Survey Department

Directorate of Minerals

Ministry of Oil

P.O. Box 2330

Aliviya, Baghdad

C Survey Department

Directorate General of Surveys

Baghdad

Director: Fuad Mohamad Ali Al-Hakim

$\mathrm{H}$ Institute of Research on Natural Resources

Abu Chraib

Baghdad

Director: Abood Al-Khafaji

R Ministry of Oil and Minerals

Baghdad

Minister: Saddoon Hammadi
NEAR EAST-SOUTH ASIA-Continued

\section{Israel}

G Geological Survey of Israel

30 Malchei Israel Street

Jerusalem

Director: Eliahu Zohar

G, H Institute for Petroleum Research and Geophysics

P.O. Box 269

Holon

Managing Director: Z. Benari

C Department of Surveys

Ministry of Labor

P.O. Box 622941

Tel Aviv

Director: R. Adler

$\mathrm{H}$ Hydrological Service

Jerusalem

$\mathrm{H}$ Tahal-Water Planning for Israel

Tel Aviv

Director General: Aaron Weiner

R Ministry of Development

38 Keren Hayesod Street

Jerusalem

Petroleum Commissioner: Moshe Ettinger

Jordan

G, C, Natural Resources Activity

$\mathrm{H}, \mathrm{R}$ Box 7

Amman

Director General: Yousef Nimry

G Royal Scientific Society

P.O. Box 6945

Amman

C Department of Lands and Surveys

P.O. Box 70

Amman

Kuwait

G Kuwait Oil Affairs Department Ahmadi 94

Al Kuwayt

$\mathrm{H} \quad$ Ministry of Electricity and Water

Al Kuwayt

Chief Engineer (Water and Gas) :

Abdullah Abdulmakzia al-Sharhan 
NEAR EAST-SOUTH ASIA-Continued

Kuwait-Continued

G Kuwait Institute for Scientific Research Hilali Street P.O. Box 12009

Al Kuwayt

Director: T. Oinomikado

R Kuwait National Petroleum Company (partly government owned)

P.O. Box 70

Al Kuwayt

Chairman: Ahmad Sayid Omar

R Ministry of Finance and Oil

Al Kuwayt

Lebanon

G Direction Generale des Travaux Publiques

Ministère des Ressources Naturelles et Energie Hydraulique

Beirut

C Institut de Géographie du Proche et Moyen Orient

Avenue de Damas

B.P. 2691

Beirut

Director: M. Le Lannou

C Service de la Topométrique

Directoire des Affaires Géographiques

Beirut

Director: Khaldoun Haidar

H Ministère des Ressources Hydrauliques et Electriques

Beirut

Director General: Mohammad Fawaz

\section{Nepal}

G Nepal Geological Survey

Lainchaur

Kathmandu

G Nepal Bureau of Mines

Lainchaur

Kathmandu

C Survey Department

Ministry of Land Reform, Agriculture, and Food

Kathmandu
NEAR EAST-SOUTH ASIA-Continued

Nepal-Continued

$\mathrm{H}$ Department of Hydrology and Meteorology

Kathmandu

Director General: Gopal Amatya

Oman

G Ministry of Agriculture, Fisheries, Petroleum, and Minerals

P.O. Box 550/551

Muscat

Minister: Sayyid Ahmad al Shan Fari

\section{Pakistan}

G Geological Survey of Pakistan

P.O. Box 15

Quetta

Director General: A. Mannan Khan

C Survey of Pakistan

Karachi

R Natural Resources Division

Ministry of Industries and Natural Resources

Rawalpindi

Director, Directorate of Oil Operations:

N. H. Qureshi

Qatar

G Qatar Petroleum Company

Doha

Saudi Arabia

G, R Ministry of Petroleum and Mineral Resources

Directorate General of Mineral

Resources

P.O. Box 345

Jiddah

Deputy Minister for Mineral Resources: Fadil K. Kabbani

C Aerial Survey Department

Ministry of Petroleum and Mineral Resources

P.O. Box 247

Riyadh

Director General: Soleman Rubaishy 
NEAR EAST-SOUTH ASIA-Continued

\section{Saudi Arabia-Continued}

C Centre for Applied Geology

P.O. Box 1744

Jiddah

Director: A. M. Al-Shanti

H Ministry of Agriculture and Water

Water Conservation Department

P.O. Box 478

Riyadh

Minister: Hassan Muhari

R Ministry of Petroleum and Mineral Resources

P.O. Box 247

Riyadh

Minister: Shaikh Ahmad Zaki Yamani

\section{Sri Lanka (Ceylon)}

G Geological Survey Department

48 Sri Jinaratana Road

Colombo 2

Director: D. B. Pattiaratchi

C Survey Department

P.O. Box 506

Colombo 5

H Water Resources Board

7 Sigiriya Gardens

Bagatelle Road

Colombo 3

Chairman: H. Hema Basnayake

\section{Syria}

G Directorate of Geological Research and Mineral Resources

Ministry of Petroleum

Fardos Street

Damascus

Director: Seifuddin Atfeh

C Service Géographique de l'Armée Damascus

\section{Turkey}

G Mineral Research and Exploration Institute of Turkey

Posta Kutusu 116

Eskisehir Yolu

Ankara

Director: Sadrettin Alpan
NEAR EAST-SOUTH ASIA-Continued

Turkey-Continued

C General Directorate of Turkish Mapping Service

Ministry of National Defense

Ankara

Director: Sukru Olcay

H State Hydraulic Works (DSI)

Ankara

R Ministry of Energy and Natural Resources

Bakanliklar

Ankara

Minister: Nezik Devres

R Petroleum Administration

Konue Sokak 37

Yenisehir, Ankara

President: Osman Tolun

\section{Yemen}

G Central Planning Organization

P.O. Box 175

San'a', Y.A.R.

Chairman: Dr. Abdul-Karim El-Eryani

Minister of Development: Mohammed Ahmed Al-Gunaid

G Office of Mineral Resources

Ministry of Public Works

San'a'

\section{EAST ASIA-PACIFIC REGION}

Australia

G Bureau of Mineral Resources

B.M.R. Building

Constitution Avenue

Canberra, A.C.T.

Acting Director: L. C. Noakes

C Department of National Development

Division of National Mapping

Box 667

P.O. Canberra City 2601

C Division of National Mapping

Department of National Development

Canberra

Acting Director: J. D. Lines 
EAST ASIA-PACIFIC REGION-Continued

Australia-Continued

$\mathrm{H}$ Department of National Development

Canberra

Director: A. I. McCutchan

R Federal Department of National

Development

Tasman House, Hobart Place

Civic Centre

Canberra, A.C.T.

Secretary: R. W. Boswell

\section{Borneo}

G Geological Survey Department

Borneo Region, Malaysia

Kuching, Sarawak

Malaysia

Director: J. B. Abjandin

\section{British Solomon Islands}

G Department of Geological Surveys

P.O. Box G24

Honiara, Guadalcanal

Director: D. Green

\section{Brunei}

G The Government Geologist

Brunei Town

C Survey Department of Brunei

Bandar Seri Degawan

\section{Burma}

G Directorate of Geological Survey and

Exploration

Ministry of Mines

Kanbe Road, Yankin P.O.

Rangoon

Director General: U Sein Myint

C, $\mathrm{H}$ Union of Burma Applied Research Institute

Kanbe Road, Yankin P.O.

Rangoon

Director General : Freddy ba Hli

C Directorate of Survey

Ministry of Agriculture and Forestry

Kaba-Aye Road

Rangoon
EAST ASIA-PACIFIC REGION-Continued

Peoples Republic of China

$\mathrm{G}, \mathrm{H}$ Institute of Geology

Department of Earth Sciences

Chinese Academy of Sciences

Peking

Chairman: Hou Te-Feng

G Institute of Geology and Paleontology

Department of Earth Sciences

Chinese Academy of Sciences

Nanking

Director: Chao Chin-ko

G Institute of Geophysics

Department of Earth Sciences

Chinese Academy of Sciences

Peking

Director: Chao Chin-Chang

G Academy of Geology

Ministry of Geology

Peking

Director: Hsu Chieh

C Institute of Geography

Department of Geography and Geology

Chinese Academy of Sciences

Peking

Chairman: Yin Chan-Hsun

C National Bureau of Geodesy and

Cartography

Ministry of Geology

Peking

Director: Ch'en Wai-ou

R Ministry of Coal Industry

Peking

R Ministry of Petroleum Industry

Peking

Republic of China (Taiwan)

G, C Geological Survey of Taiwan

P.O. Box 1001

Taichung 400

Taipei

Director: Ching-Chang Biq

G Chinese Earthquake Research Center

Academia Sinica

P.O. Box 58937

Taipei

Director: Yi-Ben Tsai 
EAST ASIA-PACIFIC REGION-Continued

Republic of China (Taiwan)-Continued

G Institute of Oceanography

National Taiwan University

Taipei

Director: T. Y. Chu

C Ministry of the Interior

Roosevelt Road

Taipei

$\mathrm{H} \quad$ Water Resources Planning Commission

Ministry of Economic Affairs

Taipei

Chairman: Fung Chung-Yue

G Chinese Petroleum Corporation

83 Chunghwa Road

Taipei

President: Jerome S. N. Hu

Fiji

G Geological Survey Department

P.O. Box 2020, Mead Road

Government Buildings

Suva

Director: J. C. Grover

C Department of Lands, Mines and Surveys

Director of Lands

Government of Fiji

Suva

Director: R. H. Ragnault

\section{Hong Kong}

G Mines Department, Branch Office Canton Road Government Offices Canton Road

Kowloon, Hong Kong

Head: J. H. Knapp

\section{Indonesia}

G Direktorat Geologi

[Geological Survey of Indonesia]

Djalan Diponegoro 57

Bandung

Director: Salman Padmanagara
EAST ASIA-PACIFIC REGION-Continued

\section{Indonesia-Continued}

G Direktorat Meteorologi dan Geofisik

Djalan Geredja Inggris 3

Djakarta

Director: M. Sukanto

C Balai Fotogrametri

[Institute of Photogrammetry]

Ministry of Defense

Djalan Gunung Sahar 90

Djakarta

Head: R. E. Beaupain

C Directorate of Land Use

Djakarta

C Army Topographic Service

Djakarta

C Markas Besar Angkatan Dorat

Direktorat Topografi

Gunung Sahari No. 90

Djakarta

H Department of Public Works

Djakarta

Secretary General: T. N. I. Dandi

Kadargan

R Ministry for Mining and Oil and Gas Affairs

Menteng Raya No. 3

Djakarta

Minister: Soemantri Bradjonegoro

\section{Japan}

G Nippon Kazan Gakkai

[Volcanological Society of Japan]

Earthquake Research Institute

University of Tokyo

I Yayoi-cho

Bunkyo-ku

Tokyo

President: T. Ishikawa

G Earthquake Research Institute

University of Tokyo

10-302, 4-1 Sakurajosui

Setagaya-Ky, Tokyo

Director: T. Rikitake 
EAST ASIA-PACIFIC REGION-Continued

Japan-Continued

G Public Works Research Institute

Ministry of Construction (includes engineering geology)

2-28-32 Honkomagome Bunkyo-ku

Tokyo

Director: Mitsuru Nagao

G, H Geological Survey of Japan, MITI

135 Hisamoto-cho

Kawasaki-shi, Kanagawa

Director: Isamu Kobayashi

C Geographical Survey Institute

Ministry of Construction

24-13, 3-Chome

Higashiyama, Meguro-Ku

Tokyo

Director: Saburo Nanbu

H Water Resources Bureau

Economic Planning Agency

Tokyo

R Mineral, Oil, and Coal Mining Bureau

Ministry of International Trade and Industry

1-3 Kasumigaseki, Chiyoda-ku

Tokyo 100

Director General: Kiyoshi Sho

\section{Khmer Republic (Cambodia)}

G Mines and Mineral Prospecting

Ministry of Industry

Phnom Penh

Commissioner: Sean Pengse

C Service Géographique du Cambodge

Phnom Penh

\section{Korea, Democratic Peoples Republic (North)}

G Geology and Geography Research Institute

Academy of Sciences

Mammoon-dong

Central District

P'yongyang

Director: Pak Sung Wook
EAST ASIA-PACIFIC REGION-Continued

Korea, Republic of (South)

G Geological and Mineral Institute of Korea

219-3, Karibong-Dong, Yongdungpo-Ku

Seoul

Director General: Joung Hwan Lee

C National Construction Research Institute

Ministry of Construction

43-1 Hwikyeong-dong

Dongdaemun-ku, Seoul

Director: Yoon Sang Ock

$\mathrm{H} \quad$ Bureau of Water Resources

Development

Ministry of Construction

Seoul

Laos

G Service Central des Mines du Laos

Ministère du Plan et de la Cooperation

B.P. 46

Vientiane

C Service Géographique Nationale

Ministère des Travaux Publics et de

Transports

B.P. 167

Vientiane

Director: Ms. Voravong

Malaysia

G Federal Geological Survey

Jalan Gurney

Kuala Lum,pur 15-01

Director: S. K. Chung

C Directorate of National Mapping

Jalan Gurney

Kuala Lumpur

R The Ministry of Lands and Mines

Jalan Gurney

Kuala Lumpur

Minister: Enche Abdul Ghafar Bin Bada 


\section{EAST ASIA-PACIFIC REGION-Continued}

\section{Mongolian People's Republic}

G Institute of Geology

Academy of Sciences

UI, Leniadom 2

Ulaanbaatar

Director: B. Luvsandandzan

G, C Institute of Geography and Permafrost Studies

Academy of Sciences

Ulaanbaatar

Director: S. H. Tsegmed

G, H Institute of Hydrology and Meteorology Academy of Sciences

Ulaanbaatar

Director: T. Myagmarjav

\section{New Caledonia}

G Service des Mines et de la Géologie

Rte. No. 1

B.P. 465

Nouméa

C Service Topographique

Territorial Administration Center

Avenue Paul Doumer

Nouméa Nouvelle

\section{New Guinea}

G Geological Survey

Department of Lands, Surveys, and Mines

Konedobu, Papua

Chief Government Geologist: S. Renwick

\section{New Hebrides}

G Geological Survey

British Residency

Port Vila

\section{New Zealand}

G Geophysics Division

Department of Scientific and Industrial Research

P.O. Box 368

Lower Hutt, Wellington 1

Director: T. Hatherton
EAST ASIA-PACIFIC REGION-Continued

New Zealand-Continued

G New Zealand Geological Survey

Department of Scientific and Industrial Research

P.O. Box 368

Lower Hutt, Wellington

Director: R. P. Suggate

C Department of Lands and Surveys

P.O. Box 8003

Government Buildings

Wellington

Director: W. S. Boyes

$\mathrm{H} \quad$ Ministry of Works

P.O. Box 12041

Wellington

R New Zealand Mines Department

Oil and Gas Exploration

Anvil House, Wakefield Street

P.O. Box 6342

Te Aro, Wellington

Chief Inspector: L. S. Jones

\section{Philippines}

G Commission on Vulcanology

University of the Philippines

Diliman, Rizal

Chairman: Emmanuel Tamesis

G Bureau of Mines

P.O. Box 1595

Herran Street

Manila

Director: Juanito Fernandez

C Coast and Geodetic Survey

University of the Philippines

Diliman, Rizal

Director: Cayeto Palma

C Board of Technical Surveys and Maps

234 Tanduay

Manila

$\mathrm{H} \quad$ Hydrology Division

Department of Public Works

Manila

\section{Singapore}

C Survey Department

Ministry of Law

Singapore 
EAST ASIA-PACIFIC REGION-Continued

Singapore-Continued

H Public Utilities Board

Chief Engineers Office

Singapore

Thailand

G, R Department of Mineral Resources Ministry of National Development Rama VI Road

Bangkok

Director General: Saman Buravas

C Thai Survey Department

Armed Forces Supreme Command

Bangkok

Deputy Director of Survey: Chumphon Kulkasem

H Mekong Committee

c/o ECAFE

Bangkok

Democratic Republic of Vietnam (North)

G Geologic Section

State Committee of Sciences

Hanoi
EAST ASIA-PACIFIC REGION-Continued

Democratic Republic of Vietnam (North)

-Continued

State Committee of Sciences

Hanoi

G Station of Oceanography

Republic of Vietnam (South)

G Institute of Oceanography

Nha-Trang

Director: Tran Ngoc Loi

G Bureau of Natural Resources

Ministry of Economy

59 Gia Long

Saigon

Director: Pham Viet Bang

C Service Géographique Nationale Ministère de la Défense Nationale Commandement et Direction du Genie Dalat

Director: Doan Van Kieu

H National Water Supply Agency 94 Cóng Ly

Saigon

Western Samoa

C Lands and Survey Department Apia 